\title{
TACO, an automated toolchain for model predictive control of building systems: implementation and verification
}

\author{
F. Jorissen, W. Boydens \& L. Helsen
}

To cite this article: F. Jorissen, W. Boydens \& L. Helsen (2018): TACO, an automated toolchain for model predictive control of building systems: implementation and verification, Journal of Building Performance Simulation, DOI: 10.1080/19401493.2018.1498537

To link to this article: https://doi.org/10.1080/19401493.2018.1498537

+ View supplementary material

曲 Published online: 09 Aug 2018.

Submit your article to this journal $\pi$

View Crossmark data 


\title{
TACO, an automated toolchain for model predictive control of building systems: implementation and verification
}

\author{
F. Jorissen (10) ${ }^{a *}$ W. Boydens ${ }^{b, c}$ and L. Helsen (10), \\ ${ }^{a}$ Department of Mechanical Engineering, University of Leuven, Leuven, Belgium ${ }^{b}$ Boydens Engineering, Dilbeek, Belgium; \\ ${ }^{c}$ Department of Architecture and Urban planning, University of Ghent, Ghent, Belgium ${ }^{d}$ EnergyVille, Genk, Belgium
}

(Received 5 February 2018; accepted 5 July 2018)

\begin{abstract}
This paper presents TACO (Toolchain for Automated Control and Optimization), which is a Modelica-based automated toolchain for model predictive control (MPC) of building systems. Its goal is to significantly reduce the engineering expertise and the time investment required for applying MPC to buildings. TACO is based on JModelica. Modifications compared to JModelica are discussed and the implementation of our custom MPC problem formulation is presented. The implementation is verified using two example models and is benchmarked with respect to accuracy and computation time. These results show that the computation time can be reduced significantly using the toolchain options, while only slightly reducing the controller optimality.
\end{abstract}

Keywords: Modelica; model predictive control; optimization; buildings; HVAC; TACO

\section{Introduction}

Building space heating and HVAC account for $15 \%$ of the world final energy use (International Energy Agency 2015) and the associated $\mathrm{CO}_{2}$ emissions have a negative impact on our climate. New building codes enforce stricter standards on the insulation of buildings and their energy performance in general, which reduces the final energy demand. Energy-efficient heat production technologies such as heat pumps, potentially combined with low-temperature heat emission systems, allow the heat conversion efficiency to increase, which further reduces the primary energy demand. Low-temperature emission systems such as floor heating or concrete core activation (CCA) increase the thermal mass of buildings. The increased thermal mass and insulation levels increase the thermal time constants of buildings. This includes the time constants of the emission system when using CCA. New buildings are therefore harder to control using rule-based control (RBC) systems that use control logic such as hysteresis controllers, PI(D)s and heating curves, which do not inherently integrate system delays and forecasts. Moreover, RBC strategies typically do not anticipate the impact of future disturbances on the current control action, or the impact of the current control action on future system states and therefore on the future system control action. This incurs an efficiency loss since thermal loads can be shifted in time when production is either more efficient or when a different heat source can be used. New buildings typically have more complex thermal systems, which have many degrees of freedom such as valve openings, pump speeds and supply temperature set points. A single degree of freedom often affects many zones of such buildings and coordinating between these different interests is hard for an RBC. This may cause conservative set points to be used in order to satisfy the zone that needs the largest control action, which can lead to an excessive control action for other zones, e.g. when using return water temperature controlled two-way valves for a set of parallel CCA circuits, an increase in the supply water temperature demand for one CCA slab, will increase the heat delivered to all CCA slabs. Secondary systems may automatically compensate for this, which can, e.g. lead to simultaneous heating and cooling of the same zone. In addition to an increase in the primary energy use of such buildings, thermal comfort may not be provided, e.g. due to overheating caused by solar gains or by insufficient secondary capacity to compensate an excessive control action of the primary system. Such problems can cause an uncomfortable environment in buildings in general, and a loss of productivity in office buildings, which has indirect financial implications (Verhelst 2017; Wargorcki et al. 2006). Badly performing RBCs can lead to long commissioning periods and possibly even to the installation of extra secondary systems. This further increases the total cost of ownership of a building, and can even lead to energyefficient devices being switched off in favour of secondary

\footnotetext{
*Corresponding author. Email: filip.jorissen@kuleuven.be
} 
systems, or a malfunction of the energy-efficient device or the controller may go unnoticed if the secondary device takes over.

This illustrates that state-of-the-art RBCs are not always capable to cope with the complexity of contemporary thermal systems in buildings. Model predictive control (MPC) is a fundamentally different type of controller, that has the potential to solve many, if not all, of the aforementioned problems. MPC is a control strategy that optimizes a system's control inputs using a computer model of the system such that a cost function is minimized. Constraints can be enforced to bound the allowed solution space of the optimization problem. MPC can be applied to building control problems. It then typically minimizes the energy use or energy cost of the building, while enforcing constraints such as technical constraints and comfort constraints, but other cost functions are possible too. MPC has already been realized for building applications. Real implementations of MPC have demonstrated energy savings of $17 \%$ in a Swiss office building (Sturzenegger et al. 2013, 2016), 17\% in a Belgian office building in Hasselt (Váňa et al. 2014), more than $30 \%$ in a Belgian office building in Brussels (De Coninck and Helsen 2016), 19\% and 27\% in two Australian office buildings (West, Ward, and Wall 2014), and 15-28\% in a Czech building (Cigler et al. 2013; Prívara et al. 2011; Široký et al. 2011), all with a similar or improved thermal comfort. Hilliard, Kavgic, and Swan (2016) present a review of recent MPC strategies. Clearly, there is a large potential for energy savings using MPC while at the same time improving thermal comfort, although the potential depends on the building type and the reference compared with (Cigler et al. 2013). Despite numerous demonstrations of MPC in buildings, commercially exploiting this potential is difficult due to the large amount of work incurred by setting up the MPC controller model (Cigler et al. 2013; Sturzenegger et al. 2014). An MPC development strategy that is both practical and scalable to large problem sizes has not been demonstrated to date. Further research is therefore required to simplify MPC controller model development, for instance, through the development of a convenient framework for setting up MPC controller models, as was also mentioned by the editorial of Henze (2013): "the question begs whether the time has come for a unified and open-source software environment for building MPC". We impose the following requirements for such a framework.

(1) Controller model development should be fast.

(2) Expert knowledge can be required for component model development, but no expert knowledge should be required for developing MPC models using combinations of these component models. That is, models should be object-oriented, such that they are generic and easy to combine by users, and easy to maintain by developers.
(3) The framework should be scalable to large problems, with reasonable computation times.

(4) The resulting MPC should be exportable and robust.

(5) The framework preferably leverages existing standards and tools such that it is future-proof.

Sturzenegger et al. (2012, 2014) presented Building Resistance-Capacitance Modelling (BRCM), a Matlab toolbox for setting up bilinear building envelope MPC models and input data using basic building construction data contained in an EnergyPlus input file. The toolbox however does not provide a generic way for modelling building HVAC equipment.

De Coninck et al. (2016) presented a toolbox for identifying the parameters of low-order 'grey-box' RC building models using building measurement data. This approach however requires measurement data of high quality with few unmodelled disturbances and large excitations and has not yet been demonstrated for multi-zone problems. The grey-box toolbox uses Modelica (Elmqvist and Mattsson 1997), an object-oriented equation-based modelling language. Although the initial scope of Modelica is to perform dynamic simulations, tools such as JModelica (Åkesson et al. 2010) and OpenModelica (Fritzson et al. 2005) also allow derivative-based optimization of such models, which can be used to implement an optimal control problem (OCP) (Bonvini and Wetter 2015) or MPC (De Coninck and Helsen 2016). These tools however use general-purpose solvers and algorithms, which do not exploit the linear nature and structure of our MPC optimization problems. This reduces computation speed and therefore scalability.

Jorissen and Helsen (2016) developed a linear automated toolchain for MPC (LAT-MPC), which uses a linear (Picard, Jorissen, and Helsen 2015) building model that is implemented using the Modelica library IDEAS (Jorissen et al. 2018). LAT-MPC only supports linear models since the model is extracted by computing the system's state space model using finite differences of the state variables and outputs with respect to the model inputs (Picard, Jorissen, and Helsen 2015). LAT-MPC was applied to an office building model that consists of 24 zones and 1330 states. The HVAC models were also linearized. Post-processing is required to map the linear HVAC model degrees of freedom (e.g. flow rates) into the system degrees of freedom (e.g. valve positions). Developing a LAT-MPC therefore still requires more optimal control and modelling expertise than what can be expected from the intended users. Moreover, the solution for the case study is suboptimal due to the conservative model approximations that were used.

These issues can be avoided by using non-linear MPC problems. JModelica implements a full Modelica parser and can thus be used to develop non-linear MPC problems. The JModelica optimization parser however does not 
support discrete decision variables or the Modelica Standard Library data reader (CombiTimeTable), which is used in the IDEAS weather data reader. Moreover, JModelica implements collocation by default, which approximates state and algebraic variables using piecewise polynomials and a fine-grained temporal discretization (Åkesson et al. 2010). Such a fine discretization is expected to result in larger computation times for multi-zone building models, which have in the order of a thousand state variables and thousands of algebraic variables.

The contribution of this paper is as follows. Due to the reasons outlined above, we present TACO, a toolchain that generates optimization code from a Modelica model implemented using libraries such as the IDEAS and the IBPSA (Annex 60) library (Wetter and van Treeck 2017). TACO is a modified version of JModelica that includes modifications that allow the use of data readers. TACO further implements a new and efficient problem formulation that exploits the near-linear nature of building dynamics, but also allows the use of steady-state non-linear HVAC models. The implementation and a verification of TACO are discussed in this paper. The main contribution is thus the development of a methodology that greatly facilitates the development of MPCs in practice. For a discussion of numerical optimization algorithms and principles we refer the reader to Nocedal and Wright (1999) and Diehl (2014).

Section 2 discusses the main algorithms of the implemented framework and the modifications that were made to the existing JModelica toolchain. Implementation details are discussed in Section 3. The algorithms are verified and benchmarked in Section 4. Finally, conclusions are presented in Section 5.

\section{Methodology}

TACO is a modified version of JModelica $v_{9608}$ that aims to provide a tool for user-friendly and low-cost MPC controller development for models that have a specific mathematical structure. This structure can be found in building models, but TACO is not limited to building models. A white-box building envelope model first needs to be developed using the IDEAS library (Jorissen et al. 2018), which has an option to linearize all time dynamics (Picard, Jorissen, and Helsen 2015) such that the building envelope model state variables have linear interdependencies only. The involved complexity and overhead for developing the model are similar to the development of a regular building energy simulation model. ${ }^{1}$ Additionally, non-linear HVAC components can be added as long as the model constraints and cost function are twice continuously differentiable $\left(C^{2}\right)$ with respect to the optimization variables. Furthermore, HVAC models should be steady state, by which we mean that they should not define state variables. However, the HVAC equations can depend non-linearly on building envelope states. An exception to this rule are HVAC models with dynamics that are not significantly faster than the control time step, such as storage tanks. They are supported as long as the time derivative of a state variable depends only linearly on that state variable. Integer optimization variables are currently not supported by JModelica.

TACO automatically transforms the Modelica model into an optimization problem of the form

$$
\min f(\mathbf{o}, \mathbf{u})
$$

subject to

$$
\begin{gathered}
\underline{\mathbf{g}}(\mathbf{u}) \leq \mathbf{g}(\mathbf{o}, \mathbf{u}) \leq \overline{\mathbf{g}}(\mathbf{u}) \\
\underline{\mathbf{o}} \leq \mathbf{0} \leq \overline{\mathbf{0}}
\end{gathered}
$$

where $\mathbf{o}$ is a vector of optimization variables with lower bounds $\underline{\mathbf{o}}$ and upper bounds $\overline{\mathbf{o}}, \mathbf{u}$ is a vector of inputs, $f(\mathbf{o}, \mathbf{u})$ is the cost function, and $\mathbf{g}(\mathbf{o}, \mathbf{u})$ is a vector of constraints with upper bounds $\overline{\mathbf{g}}(\mathbf{u})$ and lower bounds $\underline{\mathbf{g}}(\mathbf{u})$.

The objective function and constraint equations are implemented using the $\mathrm{C}++$ interface of CasADi 3.1 (Andersson et al. 2018), which computes derivative information using algorithmic differentiation (AD). In our examples, the resulting functions are optimized using IPOPT 3.12.6 (Andreas and Biegler 2006) and linear solver ma27 (HSL 2017), which are installed separately from the toolchain.

In Section 2.1, we first present at a high level how the existing JModelica functionality has been modified. Next, Section 2.2 presents the used time discretization formulation that replaces collocation. This formulation is computationally efficient since it reduces the number of required optimization variables and constraints. The remaining sections present other aspects that effect the number of optimization variables and constraints, or their sparsity: variable inlining (Section 2.3), iteration variables for non-linear expressions (Section 2.4), discrete time dynamics filtering (Section 2.5) and non-equidistant time discretizations (Section 2.6). Finally, Section 2.7 summarizes the resulting optimization problem definition of objective (1) and constraints (2).

\subsection{Toolchain structure}

We first outline the main algorithms and building blocks of the existing JModelica simulation and optimization toolchains and then discussion how these were recombined and modified in TACO.

The left part of Figure 1 summarizes the initial structure of the JModelica toolchain. A Modelica model is defined in an .mo file, which uses a number of Modelica libraries such as IDEAS and IBPSA. An optimica (Åkesson et al. 2010) .mop file extends this model and specifies cost function, optimization variables and constraints and couples them with the simulation model through equations that use variables that are declared in the simulation model. The resulting files are passed to JModelica, which parses all model 

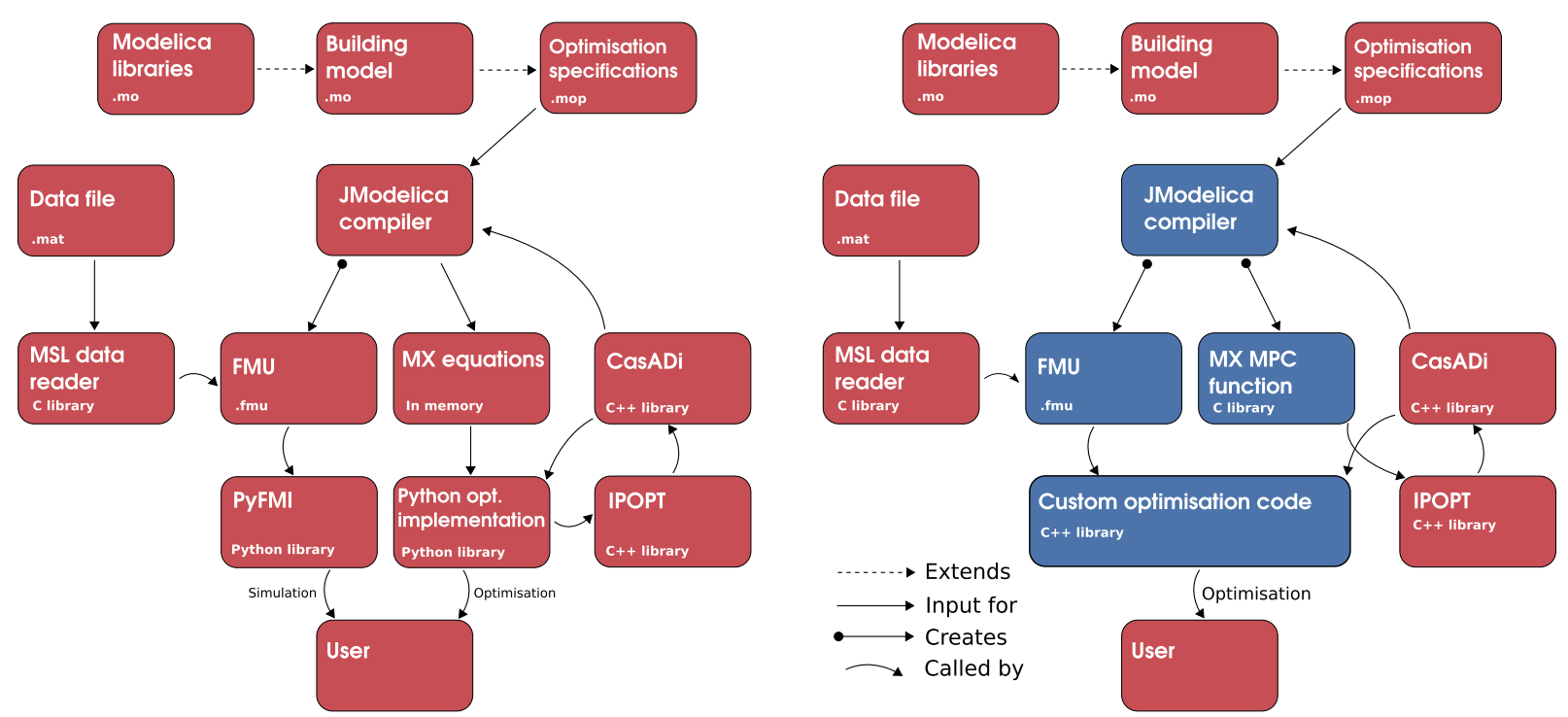

Figure 1. Schematic overview of JModelica toolchain (left) and modified toolchain (right). Modified or new blocks are indicated in blue.

files and collects all variable declarations $\mathbf{V}$ and all equations $\mathbf{E}$. These sets of equations and variables are analysed using Tarjan's algorithm (Tarjan 1972), which determines what equation groups (blocks) $\mathcal{E}$ are solved for what variable groups $\mathcal{V}$. Typically, one equation is solved for one variable, but when algebraic loops are generated, multiple equations are solved for multiple variables simultaneously. This information is collected in the BLT (block lower triangular). For simulation purposes the BLT is transformed into a functional mockup unit (FMU), which is called by the user through PyFMI. For optimization purposes the BLT equations are transformed into a CasADi MX object representation, which is used in Python to create an optimization problem using collocation and which is solved using IPOPT.

This toolchain structure is modified to the structure presented in the right part of Figure 1. The key difference is that the model equations are split into two parts. The first part contains all equations $\mathbf{U}: \mathbb{R} \rightarrow \mathbb{R}^{n_{u}}$ that depend on time only. That is, these equations and variables do not depend directly, or indirectly, on state variables or optimization variables. They are the boundary conditions of the model, which are denoted using $u(t) \in \mathbb{R}^{n_{u}}$. These equations are compiled into an FMU using parts from the existing simulation toolchain. The advantage is twofold. Firstly, we are not constrained by the type of equations that are supported by the JModelica optimization toolchain, e.g. equations can be discrete and they can use external C functions such as the MSL CombitimeTable data reader. Secondly, these equations do not need to be evaluated as part of the optimization problem, which reduces the computational overhead.

The second model part consists of the remaining equations, which is a mix of differential equations $\mathbf{I}$ :
$\mathbb{R}^{n_{x}} \times \mathbb{R}^{n_{u}} \times \mathbb{R}^{n_{z}} \times \mathbb{R}^{n_{o}} \rightarrow \mathbb{R}^{n_{x}}$ that solve for state derivatives $^{2} \mathrm{~d} x(t) / \mathrm{d} t \in \mathbb{R}^{n_{x}}$ and algebraic equations $\mathbf{H}: \mathbb{R}^{n_{x}} \times$ $\mathbb{R}^{n_{u}} \times \mathbb{R}^{n_{z}} \times \mathbb{R}^{n_{o}} \rightarrow \mathbb{R}^{n_{z}}$ that solve for algebraic variables $z(t) \in \mathbb{R}^{n_{z}}$. The model constraints $\mathbf{G}: \mathbb{R}^{n_{x}} \times \mathbb{R}^{n_{u}} \times \mathbb{R}^{n_{z}} \times$ $\mathbb{R}^{n_{o}} \rightarrow \mathbb{R}^{n_{g}}$ and the objective function integrand $J: \mathbb{R}^{n_{x}} \times$ $\mathbb{R}^{n_{u}} \times \mathbb{R}^{n_{z}} \times \mathbb{R}^{n_{o}} \rightarrow \mathbb{R}$ are also imported.

The combined set of equations results in an infinitedimensional continuous time optimization problem of the form

$$
\begin{gathered}
\min _{o(t)} \int_{t_{0}}^{t_{\infty}} J(x(t), u(t), z(t), o(t)) \mathrm{d} t, \\
\text { s.t. } \quad \frac{\mathrm{d} x(t)}{\mathrm{d} t}=\mathbf{I}(x(t), u(t), z(t), o(t)), \\
z(t)=\mathbf{H}(x(t), u(t), z(t), o(t)), \\
u(t)=\mathbf{U}(t), \\
x\left(t_{0}\right)=x_{0} \\
0 \leq \mathbf{G}(x(t), u(t), z(t), o(t)),
\end{gathered}
$$

where $x_{0} \in \mathbb{R}^{n_{x}}$ is the initial state of the system and $o(t) \in \mathbb{R}^{n_{o}}$ are the control inputs, which are declared in the Modelica model by adding the optimica attribute free $=$ true to the variable declarations. Note that this problem definition can be extended in the future, for instance, to include a cost term in (4) for the peak electrical power use during the optimized period. This continuous time problem is now discretized.

\subsection{Discrete time optimization problem}

We now describe how (4)-(9) are transformed into cost function (1), constraints (2) and optimization variable 
bounds (3). Equations (4)-(9) are infinite-dimensional and therefore the problem is discretized. The discretization method determines how many optimization variables and constraints exist in the actual optimization problem. As mentioned earlier, we do not use collocation. This is motivated by the fact that building envelope models can be simplified slightly such that they have linear dynamics. Such models can be discretized more efficiently using a custom discretization approach, which is now presented.

The cost function equals

$$
\int_{t_{0}}^{t_{\infty}} J(x(t), u(t), z(t), o(t)) \mathrm{d} t
$$

where $t_{0}$ and $t_{\infty}$ are the start and the end of the MPC horizon. We do not solve integrals analytically, since $J(x(t), u(t), z(t), o(t))$ can contain non-linear expressions, such that a solution may not be known. The cost function is therefore discretized as

$$
f(\mathbf{o}, \mathbf{u})=\sum_{i=1}^{n_{c}} \Delta t_{i} J(x[i], u[i], z[i], o[i]),
$$

where

$$
\begin{gathered}
u[i]=0.5[u(t[i-1])+u(t[i])], \\
z[i]=\mathbf{H}(x[i], u[i], z[i], o[i]),
\end{gathered}
$$

for discrete times $\left\{t[i] \in \mathbb{R}, i \in\left\{1, \ldots, n_{c}\right\}\right\}$ where $n_{c}$ is the number of control intervals and using

$$
\Delta t_{i}=t[i]-t[i-1] .
$$

Discrete state variables $x[i]$ are computed from differential Equation (5) as

$$
x[i] \equiv x(t[i])=x\left(t_{0}\right)+\int_{t_{0}}^{t[i]} \mathbf{I}(x(t), u(t), z(t), o(t)) \mathrm{d} t .
$$

The term $\mathbf{I}(\cdot, \cdot, \cdot, \cdot)$ can contain non-linear expressions. However, our building models have a specific mathematical structure with mostly linear dependencies between the building envelope state variables, such that they can be formulated as

$$
\begin{aligned}
\frac{\mathrm{d} x(t)}{\mathrm{d} t}= & \mathbf{I}(x(t), u(t), z(t), o(t))=A x(t)+B u(t) \\
& +C \mathbf{N}(x(t), u(t), z(t), o(t))+b
\end{aligned}
$$

where $A \in \mathbb{R}^{n_{x} \times n_{x}}, B \in \mathbb{R}^{n_{x} \times n_{u}}$ and $C \in \mathbb{R}^{n_{x} \times n_{n}}$ are constant matrices, $b \in \mathbb{R}^{n_{x}}$ is a constant vector, and the elements of $N: \mathbb{R}^{n_{x}} \times \mathbb{R}^{n_{u}} \times \mathbb{R}^{n_{z}} \times \mathbb{R}^{n_{o}} \rightarrow \mathbb{R}^{n_{n}}$ equal $n_{n} \in$ $\mathbb{N}$ terms of elements of $z(t)$ that are non-linear combinations of variables that depend on the states and/or time. ${ }^{3}$ In our building models, $x(t)$ typically represents temperatures, $\mathbf{N}(\cdot, \cdot, \cdot, \cdot)$ are typically heat flow rates at the interface between the building envelope and the HVAC, $u(t)$ are time-dependent boundary conditions such as the outside temperature and heat gains through windows, and $b$ are fixed boundary conditions.

Usually, MPC problems consider optimization variables $o(t)$ that form a piecewise constant trajectory as a function of time. In this work, we assume that the optimization variable values also result in a piecewise constant trajectory for $\mathbf{N}(\cdot, \cdot, \cdot, \cdot)$. This is often not true since state variable values change as a function of time and consequently $\mathbf{N}(\cdot, \cdot, \cdot, \cdot)$ changes too. This assumption could be enforced by changing the optimization variable values during a control interval, such that they counteract for the state changes during that control interval. Whether this is possible, depends on $n_{n}$ and on the number of optimization variables $n_{o}$. If $n_{n}>n_{o}$ then this leads to an overspecified problem. For this discussion, we instead assume that changes in $x(t)$ do not significantly affect the values of $\mathbf{N}(\cdot, \cdot, \cdot, \cdot)$. This assumption is verified for an example by Jorissen, Boydens, and Helsen (2018b).

If we further assume that $u(t)$ maintains a constant value $u[i]$ during interval $i$ and $z(t)$ has constant value $z[i]$, then differential Equation (5) is a constant-coefficient homogeneous first-order ordinary differential equation, which can be solved as

$$
x[i]=\hat{A} x[i-1]+\hat{B} u[i]+\hat{C} \mathbf{N}(x[i], u[i], z[i], o[i])+\hat{b},
$$

where $x[i-1]$ are the state values of previous interval and $\hat{A}, \hat{B}, \hat{C}$ and $\hat{b}$ are the discretized forms of $A, B, C$ and $b$. A derivation for these matrices is presented in Section 3.1.

Recursive definition (17) is reformulated by eliminating $x[i-1]$ from the equation such that

$$
\begin{aligned}
x[i]= & \hat{b}_{i}+\hat{A}_{i} x[0]+\sum_{j=1}^{i} \hat{B}_{i, j} u[j] \\
& +\hat{C}_{i, j} \mathbf{N}(x[j], u[j], z[j], o[i]),
\end{aligned}
$$

where $x[0]=x_{0}$ is the initial state, $\hat{A}_{i}, \hat{B}_{i, j}, \hat{C}_{i, j}$ are matrices and $\hat{b}_{i}$ are vectors. A definition for these matrices is presented in Section 3.2. Since terms $\hat{A}_{i} x[0], \hat{B}_{i, j} u[j]$ and $\hat{b}_{i}$ are functions of time intervals $i$ and $j$ only, the discrete time dynamics can be reformulated by introducing variable $u_{x}[i]$ as

$$
\begin{gathered}
x[i]=u_{x}[i]+\sum_{j=1}^{i} \hat{C}_{i, j} \mathbf{N}(x[j], u[j], z[j], o[j]), \\
u_{x}[i]=\hat{b}_{i}+\hat{A}_{i} x[0]+\sum_{j=1}^{i} \hat{B}_{i, j} u[j],
\end{gathered}
$$

where $u_{x}[i]$ are inputs of the optimization problem, which are pre-computed. This reduces the number of equations that need to be evaluated as part of the optimization problem. 
The resulting state variables $x[i]$, and $u[i]$ and $z[i]$ are used to evaluate constraint Equation (9) for each interval $i$.

\subsection{Variable inlining}

The problem formulation proposed in Section 2.1 and Section 2.2 has some advantages compared to the existing JModelica collocation implementation, which are now explained.

Firstly, building models may contain thousands of state and algebraic variables and equations. When using collocation, these equations are replicated many times, depending on the chosen collocation interval and the number of collocation points. Using our approach, algebraic equations are replicated only $n_{c}$ times. Moreover, since matrices $\hat{A}$, $\hat{B}, \hat{C}$ and $\hat{b}$ are computed off-line, the overhead for evaluating the linear differential equations is reduced. Also, intermediate algebraic variables and linear algebraic loops are eliminated from the optimization problem formulation. Our approach therefore leads to fewer equations and fewer mathematical operations.

Secondly, the proposed problem formulation computes state variables $x[i]$ using usually ${ }^{4}$ explicit Equation (17), whereas collocation corresponds to a fully implicit RungeKutta method (Åkesson et al. 2010). The explicit variable declarations can be inlined into the other equations, whereas implicit equations are often solved by declaring additional optimization variables and equality constraints that enforce the implicit equations. Explicit algebraic variable definitions can also be eliminated from the optimization problem by inlining them into each equation where the variable is used. This leads to a much denser problem formulation, but one that requires much fewer equality constraints to be enforced by the solver.

For example, an IDEAS building model that consists of $n$ zones has in the order of $n$ temperature constraints, $3 n$ control variables, $30 n$ state variables and $300 n$ unique algebraic variables. Therefore, if algebraic constraints

$$
z[i]-\mathbf{H}(x[i], u[i], z[i], o[i])=0,
$$

corresponding to algebraic Equation (6) were added to the optimization problem constraints $\mathbf{g}(\mathbf{o}, \mathbf{u})$, then there would exist two orders of magnitude more constraints. Moreover, since $z[i]$ would become optimization variables, there would also be two orders of magnitude more optimization variables.

For this reason, algebraic variables are inlined when they can be evaluated explicitly. The same argument can be made for the state variables. Therefore, state variables are also inlined when an explicit formulation exists.

Variables are inlined using the BLT information, which specifies what equation is solved for what variable. A variable that is explicitly solved from one equation, is inlined into each equation that uses the solved variable. However, there are two cases where an explicit solution is not available.

Firstly, when $\mathbf{H}(\cdot, \cdot, \cdot, \cdot)$ contains algebraic loops, then algebraic loop with index $l \in\left\{1, \ldots, n_{a}\right\}$ simultaneously solves a set of variables $\mathbf{V}_{l} \in \mathbb{R}^{n_{a, l}}$ from a set of equations $\mathbf{E}_{l}\left(\mathbf{V}_{l}\right): \mathbb{R}^{n_{a, l}} \rightarrow \mathbb{R}^{n_{a, l}}$, where $n_{a}$ is the number of algebraic loops and $n_{a, l}$ is the number of algebraic variables contained by algebraic loop $l$. We then cannot derive analytic expressions that compute $\mathbf{V}_{l}$ explicitly. The resulting algebraic loop can then either be solved using an iterative numerical method such as a Newton solver during each optimization iteration, or by introducing additional optimization variables $\mathbf{V}_{l}$ and additional equality constraints $\mathbf{E}_{l}\left(\mathbf{V}_{l}\right)$ that ensure that the model equations are satisfied. Our current implementation uses the second approach.

Secondly, the $m$-th element of (17) is considered an implicit equation if its term $\mathbf{N}(x[i], u[i], z[i], o[i])$ depends on any element of $x[i]$. For each of the $n_{s}$ state variables where this occurs, optimization variable $o_{s}^{(m)}[i] \in \mathbb{R}$ is added and the corresponding element of (19) is added as an equality constraint. This could be avoided by using $x[i-1]$ for the evaluation of the right hand side of (19). However, for systems with dynamics that are faster than the control time step, this is expected to lead to large errors since the response of the system on the control action is then not taken into account.

Despite that optimization variables are introduced to represent state variables, we categorize the problem as a single shooting approach since each state depends on the initial state. A weak point of single shooting is that unstable systems are difficult to treat (Diehl et al. 2006). However, building dynamics are stable.

\subsection{Iteration variables for non-linear expressions}

We explained earlier that inlining variables reduces the number of equality constraints and optimization variables. However, inlining variables significantly increases the computation time required by CasADi to evaluate the constraint Jacobian and the Hessian of the Lagrangian, especially for large problems. This could be related to the graph-colouring (Gebremedhin, Manne, and Pothen 2005) technique that is used by CasADi. The increased computation time causes the presented approach to be orders of magnitude slower for large problems, unless additional optimization variables $o_{n}[i] \in \mathbb{R}^{n_{n}}$ and equality constraints

$$
o_{n}[i]=\mathbf{N}(x[i], u[i], z[i], o[i])
$$

are introduced. In what follows, $\mathbf{N}(x[i], u[i], z[i], o[i])$ is substituted by $o_{n}[i]$ in (19).

\subsection{Discrete time dynamics filtering}

We allow matrices $\hat{C}_{i, j}$ in the discretized state Equation (19) to be 'filtered' such that the model sparsity increases. 
Element $\hat{C}_{i, j}(k, l)$ indicates how much variable $o_{n}[j](l)$ contributes to the value of state $x[i](k)$. Recall now that $\hat{C}_{i, j}$ originates in the time integration from the building dynamics. The relative importance of these contributions therefore highly depends on the 'distance' between $x[i](k)$ and $o_{n}[j](l)$ in both time and location. More specifically, (1) control actions that have occurred a long time ago, may no longer significantly influence the current state, and (2) state variables in one room of the building may not be influenced significantly by a control action at the other side of the building. Most elements of $\hat{C}_{i, j}(k, l)$ are therefore small, indicating that their influence is negligible. We therefore allow such small values to be filtered automatically through the definition of the cut-off fraction $\delta$. Element $\hat{C}_{i, j}(k, l)$ is set to zero if

$$
\operatorname{abs}\left(v(l) \hat{C}_{i, j}(k, l)\right)<\delta \max \left(\operatorname{abs}\left[v \star \hat{C}_{i, j}(k,:)\right]\right),
$$

where $\hat{C}_{i, j}(k,:)$ is row $k$ of $\hat{C}_{i, j}, v \in \mathbb{R}^{n_{n}}$ is the nominal value of $o_{n}[i]$ and $\star$ is the component-wise product.

\subsection{Non-equidistant time discretisation}

The discussion thus far implicitly assumes that constraints are enforced at the end of each of the $n_{c}$ time intervals. Such a high resolution may however not be required towards the end of the time horizon for typical MPC applications. The implementation is therefore generalized. Assuming a discrete time interval $l_{c}=\min \left(\Delta t_{i}\right)$ of 1 hour, the user must define an integer vector that indicates how many hours each control interval $\Delta t_{i}$ lasts. The optimal control variables are maintained constant during each control interval and the constraints are only enforced at the end of each control interval. The number of control variables and constraints can therefore be reduced significantly, or the MPC horizon can be extended using the same number of optimization variables. This approach is often called move blocking, the reader is referred to Cagienard et al. (2004) for more information.

\subsection{Optimization problem summary}

We now summarize the resulting computation sequence. Cost function (1) and constraints (2) are evaluated from time variable $t$ and optimization variables

$$
\begin{aligned}
\mathbf{0}=\left\{o[i], o_{a}[i], o_{s}[i], o_{n}[i]\right\}, \quad \forall i \in\left\{1, \ldots, n_{c}\right\} \\
o_{a}[i]=\left\{o_{a}^{(l)}[i]\right\}, \quad \forall l \in\left\{1, \ldots, n_{a}\right\} \\
o_{s}[i]=\left\{o_{s}^{(m)}[i]\right\} \quad \forall m \in\left\{1, \ldots, n_{s}\right\}
\end{aligned}
$$

as follows.

Firstly, initial states $x[0]$ are fetched from the FMU for the first MPC solution, and are otherwise updated using a state estimation method, which is not yet included in the toolchain. Secondly, inputs $u[i]$ are computed using the FMU for the time horizon of interest using

$$
\begin{array}{rlrl}
u(t) & =\mathbf{U}(t), & & (7 \text { revisited }) \\
u[i] & =0.5[u(t[i-1])+u(t[i])], & (12 \text { revisited })
\end{array}
$$

which are in turn used to evaluate

$$
u_{x}[i]=\hat{b}_{i}+\hat{A}_{i} x[0]+\sum_{j=1}^{i} \hat{B}_{i, j} u[j], \quad(20 \text { revisited })
$$

using pre-computed matrices $\hat{A}_{i}, \hat{B}_{i, j}$ and vector $\hat{b}_{i}$. This results in the following inputs for the optimization problem

$$
\mathbf{u}=\left\{u[i], x[0], u_{x}[i]\right\} \quad \forall i \in\left\{1, \ldots, n_{c}\right\} .
$$

Using these fixed inputs and the optimization variables, the discrete optimization problem with inlined variables $x[i]$ and $z[i]$ is formulated as

$$
\min _{\mathbf{0}} \sum_{i=1}^{n_{c}} \Delta t_{i} J^{\prime}\left(o_{s}[i], u[i], o_{a}[i], o[i]\right)
$$

$$
\text { s.t. } \quad o_{s}[i]=u_{x}[i]+\sum_{j=1}^{i} \hat{C}_{i, j} o_{n}[j], \quad \forall i \in\left\{1, \ldots, n_{c}\right\}
$$

$$
\begin{aligned}
& o_{n}[i]=\mathbf{N}^{\prime}\left(o_{s}[i], u[i], o_{a}[i], o[i]\right), \forall i \in\left\{1, \ldots, n_{c}\right\} \\
& o_{a}[i]=\mathbf{H}^{\prime}\left(o_{s}[i], u[i], o_{a}[i], o[i]\right), \forall i \in\left\{1, \ldots, n_{c}\right\} \\
& 0 \leq \mathbf{G}^{\prime}\left(o_{s}[i], u[i], o_{a}[i], o[i]\right), \quad \forall i \in\left\{1, \ldots, n_{c}\right\}
\end{aligned}
$$

where $\mathbf{H}^{\prime}(\cdot, \cdot, \cdot, \cdot)$ is the inlined version of $\mathbf{H}(\cdot, \cdot, \cdot, \cdot)$, which has fewer inputs and fewer outputs since many algebraic variables are inlined. Consequently, $J^{\prime}(\cdot, \cdot, \cdot, \cdot), \mathbf{N}^{\prime}(\cdot, \cdot, \cdot, \cdot)$ and $\mathbf{G}^{\prime}(\cdot, \cdot, \cdot, \cdot)$ also have fewer inputs.

Optimization variable bounds for inequalities (3) are obtained from the Modelica min and max attributes of the optimization variable declarations. When an attribute is not defined then positive or negative infinity is used.

The resulting set of equations is converted into CasADi MX expressions using the JModelica toolchain. CasADi detects common sub-expressions such that recurring subexpressions and inlined variables are evaluated only once. Moreover, since all equations are inlined, variables and equations that are not required for evaluating the cost function or constraints, are not evaluated since they do not appear in the objective or constraints. Therefore, only a fraction of the state variables needs to be evaluated, which reduces computation time. Self-contained C code is generated from this problem formulation using the CasADi code generator. The resulting files are compiled into a library that implements the cost function, constraints and the required derivatives as a function of $\mathbf{o}$ and $\mathbf{u}$. 


\section{Implementation details}

This section provides further details about how the toolchain has been practically implemented.

\subsection{Computation of $\hat{A}, \hat{B}, \hat{C}$ and $\hat{b}$}

Explicit Euler integration ${ }^{5}$ is used to compute $\hat{A}, \hat{B}, \hat{C}$ and $\hat{b}$ from $A, B, C$ and $b$, which are in turn derived by separating the model equations based on their dependencies on time or state variables. The matrices are then computed numerically using forward finite differences. For generality, consider that $B, C$ and $b$ are merged into a single matrix $D$ such that (16) equals

$$
\frac{\mathrm{d} x(t)}{\mathrm{d} t}=A x(t)+D c[i]
$$

where $c[i]$ is the concatenation of $u[i], n[i] \equiv n(t[i])$ and 1. Using explicit Euler integration, the values of $x(t)$ at times $t_{0}+\Delta t_{e}$ and $t_{0}+2 \Delta t_{e}$ for some $\Delta t_{e} \in \mathbb{R}$ then approximately equal

$$
\begin{gathered}
x\left(t_{0}+\Delta t_{e}\right)=x\left(t_{0}\right)+\Delta t_{e}\left[A x\left(t_{0}\right)+D c[i]\right] \\
=\left[I+\Delta t_{e} A\right] x\left(t_{0}\right)+\Delta t_{e} D c[i] \\
x\left(t_{0}+2 \Delta t_{e}\right)=x\left(t_{0}+\Delta t_{e}\right)+\Delta t_{e}\left[A x\left(t_{0}+\Delta t_{e}\right)+D c[i]\right] \\
=\left[I+\Delta t_{e} A\right]^{2} x\left(t_{0}\right)+\left[\Delta t_{e} D+\Delta t_{e}\left(I+\Delta t_{e} A\right) D\right] c[i] .
\end{gathered}
$$

For $t=t_{0}+n \Delta t_{e}$ this is generalized into

$$
\begin{gathered}
\hat{A}=\left(I+\Delta t_{e} A\right)^{n}, \\
\hat{D}=\sum_{i=0}^{n-1} \Delta t_{e}\left(I+\Delta t_{e} A\right)^{i} D .
\end{gathered}
$$

These expressions are used to compute $\hat{A}, \hat{B}, \hat{C}$ and $\hat{b}$ with $\Delta t_{e}=1 \mathrm{~s}$ and a user-defined value for the control interval length $l_{c}=n \Delta t_{e}$. To avoid large computation times, the matrix power in (38) is evaluated using exponentiation by squaring, which requires $\mathcal{O}(\log (n))$ times the cost of a matrix multiplication instead of $\mathcal{O}(n)$. Furthermore, sum (39) implies that

$$
\left.\hat{D}\right|_{n=2 j}=\left.\hat{D}\right|_{n=j}+\left.\left(I+\Delta t_{e} A\right)^{j} \hat{D}\right|_{n=j} .
$$

This sequence is used to evaluate $\hat{B}, \hat{C}$ and $\hat{b}$, also using $\mathcal{O}(\log (n))$ operations.

\subsection{Computation of $\hat{A}_{i}, \hat{B}_{i, j}, \hat{C}_{i, j}$ and $\hat{b}_{i}$}

The equality of (17) and (18) implies that

$$
\begin{gathered}
\hat{A}_{i}=\hat{A}^{i}, \\
\hat{B}_{i, j}=\hat{A}^{i-j} \hat{B}, \\
\hat{C}_{i, j}=\hat{A}^{i-j} \hat{C}, \\
\hat{b}_{i}=\sum_{j=0}^{i-1} \hat{A}^{i-j} \hat{b} .
\end{gathered}
$$

\section{Verification and benchmarks}

The implementation of TACO is now verified and benchmarked using two example models to assess the scalability. The models are described in Section 4.1. Section 4.2 presents a verification of the implementation of the discretized time dynamics. The purpose of Sections 4.3, 4.4 and 4.5 is to demonstrate the influence of some of the main parameters of the toolchain and to verify the implementation for some simple examples. For a real demonstration and discussion of the optimal control results that are obtained for a realistic building model, we refer to Jorissen, Boydens, and Helsen (2018b).

\subsection{Example model description}

Two examples are used to demonstrate the TACO, and to verify its implementation.

Example 1 is the IDEAS implementation of the BESTEST case 900 building. It consists of a single zone of $8 \mathrm{~m} \times 6 \mathrm{~m} \times 2.7 \mathrm{~m}$ and has a single south-oriented window of $12 \mathrm{~m}^{2}$. All radiative and convective heat transfer equations are linearized as described by Picard, Jorissen, and Helsen (2015) since they would otherwise introduce non-linear dynamics, and the default moist air medium is replaced by a dry air medium. A weather data set is used that has a resolution of 2 minutes. These weather data points are linearly interpolated when evaluating (12). A heat flow rate is injected in the zone air node. This heat flow rate is minimized and is lower bounded by zero. The zone air temperature is constrained to be larger than $21.85^{\circ} \mathrm{C}(295 \mathrm{~K})$. The resulting model has one optimization variable $o(t), 29$ state variables $x(t)$ of which only the air temperature is used and 22 time-dependent inputs in $\mathbf{u}$.

Example 2 is one floor of the Solarwind model (Jorissen, Boydens, and Helsen 2018a), which consists of 8 zones of which 6 are conditioned, 6 CCA slabs for which the two-way valve openings are optimized and 6 Variable Air Volumes (VAVs) for which the control signals and heating coil valve openings are optimized. The objective is to minimize the total heat flow rate supplied to the heating coils and the CCA slabs. The zone air temperature is constrained between $21.85^{\circ} \mathrm{C}$ and $23.85^{\circ} \mathrm{C}$. The resulting 
model has 18 optimization variables $o(t), 250$ states $x(t)$ of which 12 are used, 171 inputs $u(t), 12$ state iteration variables $o_{s}(t), 0$ algebraic loop iteration variables $o_{a}(t)$ and 24 iteration variables $o_{n}(t)$.

These examples are translated using TACO, which generates a library that exposes the required optimization functions. This library is called from a $\mathrm{C}++$ interface, which is in turn called periodically from Dymola 2018. This way the MPC code can be run as a $\mathrm{C}$ function in a Modelica simulation together with the actual model. That is, the optimal control results of the example model are applied to an instance of the same model in Dymola. The optimization problem is re-evaluated after each control interval. The initial guess value of all optimization variables is $10^{-5}$ for the first MPC solution. For the next MPC solutions, the optimization problem is warm-started using the converged solution of the previous optimization.

\subsection{Numerical time integration}

Example 1 is first used to verify the numerical integration implementation of Equations (19)-(20). The simulation model is therefore simulated using Euler integration with a fixed time step of $1 \mathrm{~s}$, to avoid mismatch with the integration algorithm used by the MPC code. The MPC's discrete internal variable for the air temperature $T_{\text {air,mpc }}(t) \equiv T_{\text {air,mpc }}(t[1])$ is compared to $T_{\text {air,sim }}(t)$, the simulated air temperature computed by Dymola. Note that $T_{\text {air,mpc }}(t)$ is only updated every control interval. The normalized error is computed for various values of the control interval length $l_{c}$ as

$$
e=\frac{\int_{t_{0}}^{t_{\infty}} \operatorname{abs}\left(T_{\text {air,mpc }}(t)-T_{\text {air,sim }}(t)\right) \mathrm{d} t}{t_{\infty}-t_{0}}
$$

for initial time $t_{0}=1.42 \cdot 10^{9} \mathrm{~s}$ and end time $t_{\infty}=1.43$. $10^{9} \mathrm{~s}$, which corresponds to a duration of 116 days.

Since all model equations are linear, the implementation has no model mismatch except for the discretization of the time-dependent inputs $\mathbf{u}$, which are assumed constant during each control interval. This discretization error decreases as $l_{c}$ decreases such that $e$ is expected to go to zero as $l_{c}$ goes to zero. Figure 2 illustrates that this is indeed the case. Note that the reported errors are open-loop errors, i.e. no state update is performed such that errors accumulate over time and only dissipate at a rate determined by the time constant of the building.

\subsection{Impact of MPC horizon length}

Example 1 is now used to investigate the impact of the number of control intervals $n_{c}$, i.e. the MPC horizon length, on the performance of the MPC controller. When the horizon length is longer, the MPC controller can anticipate further into the future, which should reduce the cost

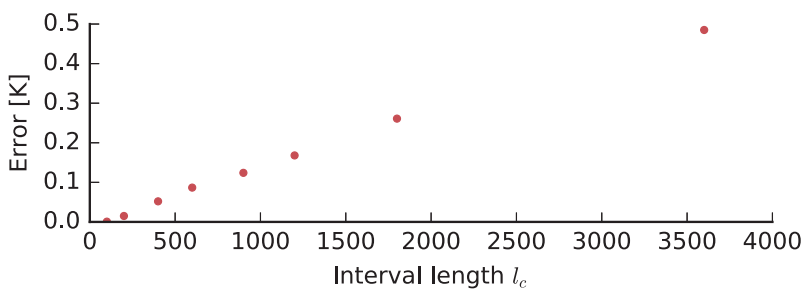

Figure 2. Open-loop optimization errors $e$ (see (45)). The error goes to zero as $l_{c}$ goes to zero.

function. We use $n_{c}$ control intervals of $l_{c}=600 \mathrm{~s}$ such that the model mismatch is in the order of $0.1 \mathrm{~K}$ (see Figure 2).

The integral of the objective $J$ is evaluated for various values $n_{c}$. The resulting objective value differences are smaller than $0.01 \%$. This result suggests that there is no relation between $J$ and $n_{c}$. Indeed, in this example no gains can be obtained by looking further into the future than 1 control interval. We qualitatively explain this by the fact that constraints do not require to pre-heat the building and the objective function does not promote this either. Constraints do not impose that the building is pre-heated since there is no upper bound for the injected thermal power. The objective does not promote pre-heating since each injected unit of energy has the same cost, and energy that is injected earlier, has more time to dissipate such that its effect on the zone air temperature is smaller. We here implicitly assume that the influence of a unit of energy added at time $t_{0}$ on the zone air temperature is a strictly decreasing function of time $t>t_{0}$. This is the case when heat is injected directly into the air node.

We therefore adjust the model such that heat is injected in the radiative node, which spreads the thermal power over all walls, floor and ceiling. Moreover, the objective now equals the square of the thermal power. For this example, the influence of the control interval length is shown in the top plot of Figure 3, where thermal power profiles for 2, 36 and 72 intervals of 10 minutes each are shown. These simulation results are generated using a Dymola simulation model that is coupled to the MPC controller generated using TACO. The MPC controller is generated from the exact same model equations as the simulation model.

The longer horizon causes the controller to pre-heat the building such that the rising slopes of the thermal power are smoother and peaks may be smaller. Decreasing slopes are in general not smoother, since smoothing two consecutive intervals would imply that the (larger) thermal power during the first interval is reduced and/or thermal power during the second interval is increased. This would incur a comfort violation at the end of the first control interval, since the thermal power is reduced.

\subsection{Impact of MPC horizon resolution}

These results illustrate the advantage of having long MPC horizons, depending on the optimization problem 

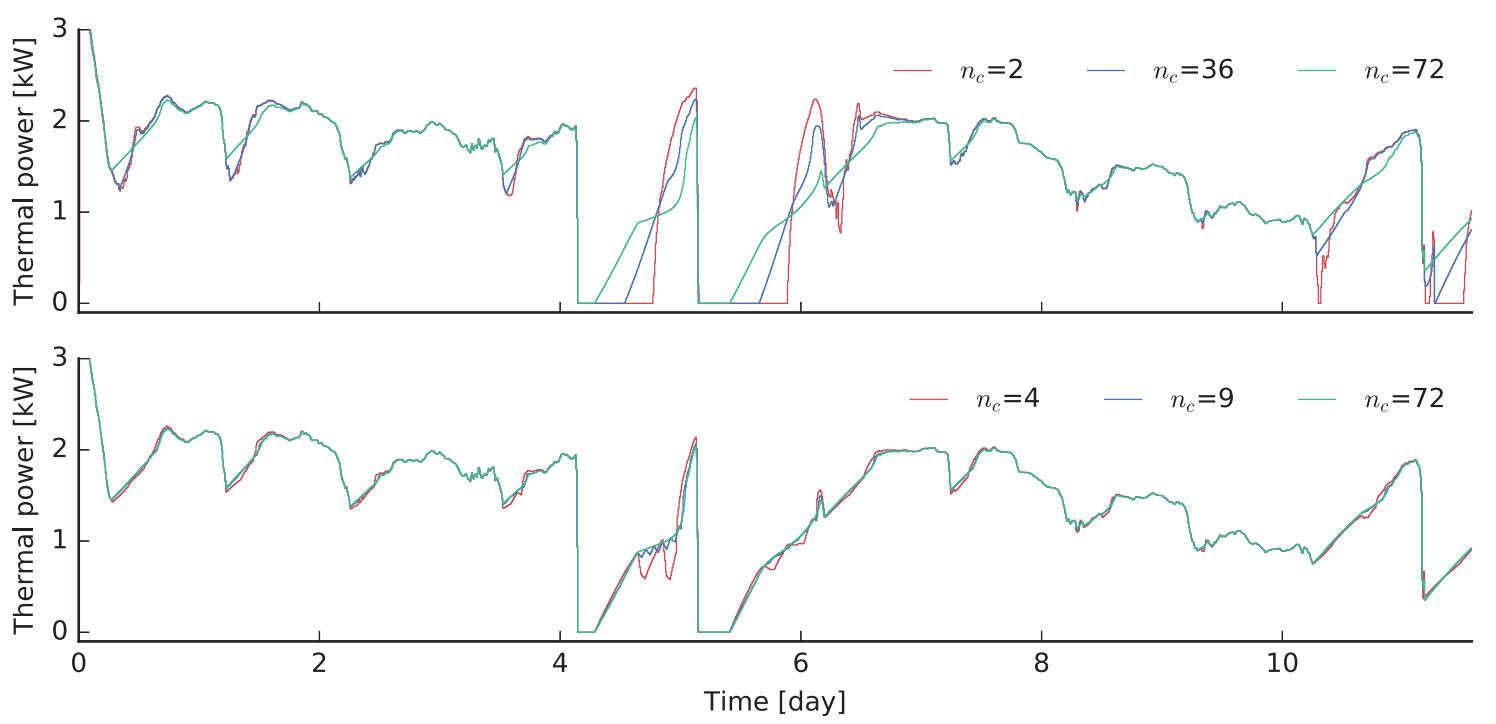

Figure 3. Optimal control results for $n_{c}=\{2,36,72\}$ control intervals of 10 minutes each (top) and for 72 intervals of 10 minutes, 9 concatenated intervals or 4 concatenated intervals.

properties. The number of optimization variables and constraints however both increase linearly with the number of optimization intervals. This results in a computation time that rises more than linearly with the number of optimization intervals, typically also in an increased number of iterations, and possibly even in convergence problems for large models. Therefore, TACO allows concatenating multiple control intervals into a single, longer control interval, as described in Section 2.6, e.g. the horizon of $n_{c}=72$ control intervals of duration $l_{c}$ can be reformulated into $n_{c}=9$ control intervals with a duration of $\{1,1,10,10,10,10,10,10,10\}$ times $l_{c}$. This results in a reduction from $n_{c}=72$ into $n_{c}=9$ sets of optimization variables and constraints, while the total horizon length stays the same. The intervals can be concatenated further, e.g. by using $n_{c}=4$ intervals with a duration of $\{1,11,30,30\}$ times $l_{c}$. The results for $n_{c}=72, n_{c}=9$ and $n_{c}=4$ are summarized in the bottom plot of Figure 3. The optimal control results are often very similar, although some clear differences can be seen starting around day 4.6. Significant drops are observed in the optimal control results, especially when 30 intervals are concatenated. The reason is a large peak in solar irradiation, which suddenly causes heat demand to be zero around day 5.1, which is 720 minutes later. At day 4.6, the last temperature constraint of the MPC controller is enforced before the end of the peak power requirement. During the next control interval, the constraint is enforced after the peak power requirement and the MPC controller does not see the peak demand just before that. The power demand for smoothly pre-heating at the current time step can therefore be reduced, since the power demand in the future is also reduced. This is observed as a dip in the optimal control result. The optimal control result then recovers as the second last constraint nears the peak, after which it drops again. Note that although the results are visually different, the integral of the objective changes only slightly from $2.55671 \cdot 10^{12} \mathrm{~J}$ for $n_{c}=72$ to $2.55721 \cdot 10^{12} \mathrm{~J}$ for $n_{c}=9$ and $2.56126 \cdot 10^{12} \mathrm{~J}$ for $n_{c}=4$ for this example.

The impact of the concatenations on the computation time cannot be measured sufficiently accurately for Example 1 because of the small model size. The same analysis is therefore performed using Example 2.

Table 1 summarizes the main computation times for this model as reported by CasADi. The simulations are performed for a case with and without iteration variables $o_{n}[i]$. These results show that the computation times for both the Jacobian and the solver scale more than quadratically with $n_{c}$ for sufficiently large values of $n_{c}$, and illustrate the effect of the use of the iteration variables for $o_{n}[i]$. The solver time of IPOPT decreases, despite the larger number of optimization variables, when adding iteration variables $o_{n}[i]$. A likely cause is that the equality constraint Jacobian has only 604,416 instead of 748,800 non-zero entries when using iteration variables for $n_{c}=64$. The respective constraint Jacobians have $7.6 \%$ and $51 \%$ non-zero entries. These results further show that the evaluation time when using the iteration variables is faster. On average the Jacobian evaluation time scales as $\mathcal{O}\left(n_{c}^{2.1}\right)$ and $\mathcal{O}\left(n_{c}^{1.86}\right)$ for the

Table 1. Computation times for Example 2 per evaluation of the constraint Jacobian $t_{\mathrm{jac}}$ and per iteration of the solver (IPOPT) $t_{s o l}$ for different values of the number of control intervals $n_{c}$.

\begin{tabular}{llllllrc}
\hline & with & & & & & & \\
$n_{c}$ & $o_{n}[i]$ & 2 & 4 & 8 & 16 & 32 & 64 \\
\hline$t_{\text {jac }}[\mathrm{ms}]$ & no & 0.45 & 1.7 & 9.9 & 36.9 & 143 & 566 \\
$t_{\text {sol }[\mathrm{ms}]}$ & no & 0.5 & 0.8 & 2.8 & 12.8 & 71 & 499 \\
$t_{\text {jac }}[\mathrm{ms}]$ & yes & 0.04 & 0.08 & 0.18 & 0.58 & 2.6 & 8.7 \\
$t_{\text {sol }[\mathrm{ms}]}$ & yes & 0.6 & 0.9 & 2.1 & 9.8 & 51 & 350 \\
\hline
\end{tabular}


Table 2. Computation times for Example 2 per evaluation of the constraint Jacobian $t_{\mathrm{jac}}$ and per iteration of the solver (IPOPT) $t_{\mathrm{sol}}$ for different control interval lengths, with iteration variables $o_{n}[i]$.

\begin{tabular}{llllc}
\hline$n_{c}$ & 4 & \multicolumn{1}{c}{8} & 16 & 64 \\
\hline$t_{\mathrm{jac}}[\mathrm{ms}]$ & 0.08 & 0.17 & 0.57 & 8.7 \\
$t_{\text {sol }}[\mathrm{ms}]$ & 1.0 & 2.0 & 9.6 & 350 \\
\hline
\end{tabular}

case without and with iteration variables. In both cases the solver time scales as $\mathcal{O}\left(n_{c}^{2.5}\right)$.

Table 2 summarizes the dominating computation times of the optimization problem for the same model when control intervals are concatenated using interval lengths

- $\{1,3,30,30\}\left(n_{c}=4\right)$,

- $\{1,3,10,10,10,10,10,10\}\left(n_{c}=8\right)$,

- $\{1,3,4,4,4,4,4,4,4,4,4,4,5,5,5,5\}\left(n_{c}=16\right)$ and

- 64 intervals of duration $1\left(n_{c}=64\right)$.

These results illustrate that the computation time can be reduced significantly by concatenating intervals. Note that the computation times do not increase relative to the results in Table 1 . Indeed, the equation structure for $n_{c}$ intervals does not depend on the length of each interval. Only the numerical values of the non-zero coefficients in $\hat{C}_{i, j}$ and the values of $u_{x}[i]$ are affected.

\subsection{Impact of model sparsity}

The results in Table 1 and Table 2 show that the computation time of the solver dominates the computation time of the derivatives. Significant speedups can therefore only be obtained by improving the solver speed. Table 1 already illustrates that the Jacobian sparsity can have an influence on the solver speed. The constraint Jacobian sparsity highly depends on matrices $\hat{C}_{i, j}$ in (19) since this matrix links state-dependent constraints to optimization variables $o_{n}[i]$. Sparser matrices $\hat{C}_{i, j}$ lead to a sparser constraint Jacobian. We therefore attempt to increase the sparsity of $\hat{C}_{i, j}$ using the filtering approach presented in Section 2.5.

Table 3 shows the influence of this option for the second example using multiple values of $\delta$ and control interval lengths $\{1,3,10,10,10,10,10,10\}$. The number of non-zero entries in $\hat{C}_{i, j}$ can be reduced by $91 \%$ when filtered using $\delta=10^{-2}$ and the computation time is then reduced by about $90 \%$. Note that the values of $t_{\text {jac }}$ are integer multiples of $0.14 \mathrm{~ms}$, where the integer multiplier equals the number of files over which the $\mathrm{C}$ code is split. This code could therefore potentially be implemented more efficiently. Moreover, $t_{\text {sol }}$ decreases most significantly between $\delta=0$ and $\delta=10^{-10}$ despite that only $20 \%$ of the non-zero coefficients are removed. This may be caused by filtering small coefficients that make the problem numerically ill-posed.

The resulting code is tested in an open-loop MPC simulation of 277 hours using $l_{c}=3600$; s for $\delta=0, \delta=10^{-4}$, $\delta=10^{-3}, \delta=10^{-2}$. In all cases the comfort violations are in the order of $0.1 \mathrm{~K}$ and the objective value changes less than $1 \%$. These simplifications therefore do not have a significant impact on the results for Example 2.

\section{Conclusion}

This paper demonstrates TACO, a JModelica-based toolchain for the development of MPC for building systems and verifies its implementation. Its goal is to significantly reduce the engineering cost and expertise required for MPC development, through the use of detailed, objectoriented Modelica models that encapsulate the model complexity. For the building envelope, Modelica models from the IDEAS library can be used directly, without the need for model order reduction. For the HVAC systems, detailed Modelica models can be used. The need for post-processing is avoided by directly mapping control variables of the system to optimization variables. However, integer decision variables are currently not supported.

Changes to the structure of the existing JModelica toolchain are elaborated, and the main toolchain algorithms and important equations are explained. Our implementation is computationally efficient, since (1) time-dependent inputs of the optimization problem are pre-computed using an FMU, which reduces the optimization problem size, (2) the collocation algorithm of JModelica is replaced by a single shooting approach that efficiently pre-computes the linear part of the thermal dynamics, (3) we implemented a 'filter' that removes the dependency of constraints with respect to optimization variables to which they have a small sensitivity, which increases the model sparsity and (4) we implemented move blocking, which reduces the number of optimization variables and constraints while keeping the same MPC horizon length. Building envelope

Table 3. Computation times per evaluation of the constraint Jacobian $t_{\mathrm{jac}}$ and per iteration of the solver (IPOPT) $t_{\text {sol }}$ for different cut-off filters that set fraction $f$ of the non-zero entries in the set of $\hat{C}_{i, j}$ matrices to zero.

\begin{tabular}{lllllllllll}
\hline$\delta$ & 0 & $10^{-10}$ & $10^{-9}$ & $10^{-8}$ & $10^{-7}$ & $10^{-6}$ & $10^{-5}$ & $10^{-4}$ & $10^{-3}$ & $10^{-2}$ \\
\hline$t_{\text {jac }[\mathrm{ms}]}$ & 0.57 & 0.28 & 0.28 & 0.28 & 0.27 & 0.27 & 0.15 & 0.15 & 0.14 & 0.14 \\
$t_{\text {sol }[\mathrm{ms}]}$ & 9.6 & 2.2 & 2.1 & 2.0 & 1.8 & 1.4 & 1.2 & 1.0 & 0.7 & 0.7 \\
$f[\%]$ & 0 & 20 & 26 & 34 & 42 & 54 & 64 & 75 & 86 & 91 \\
\hline
\end{tabular}


model dynamics must be linear and time-independent, such that the model dynamics can be pre-computed. This constraint is satisfied for our building application since IDEAS models are parameterized to be linear time-independent. Steady-state non-linear HVAC equations, constraints and objective are allowed. TACO supports the use of data readers and supports code export for the resulting MPC controller. Our implementation is verified using a first (simple) example model that demonstrates that the integration error of a zone temperature goes to zero as the control interval length goes to zero. Furthermore, the impact of the MPC horizon length and horizon resolution on the computation time and objective value are demonstrated using a second (more complex) example model. Finally, we demonstrate the impact of our filtering technique on the model sparsity and the corresponding computation time.

A companion paper applies the TACO framework to a multi-zone office building model (Jorissen, Boydens, and Helsen 2018a), which demonstrates the performance of the framework and the energy savings potential of optimal control of advanced HVAC systems. Furthermore, it is shown that TACO efficiently scales to large problem sizes, and to complex non-linear systems (Jorissen, Boydens, and Helsen 2018b). TACO will become available once licensing issues have been resolved.

\section{Notes}

1. This overhead could however be reduced through the use of templates or automated toolchains based on building information modelling.

2. We here implicitly assume that the equations can be solved explicitly for the state derivatives.

3. For example, the square of a state variable, or the multiplication of a state variable and an element of $u(t)$ are included in $\mathbf{N}(\cdot, \cdot, \cdot, \cdot)$, but the product of a state variable and a constant is included in matrix $A$.

4. The equation is usually explicit, but not always, see further in this section.

5. Note that matrix exponentials could have been used instead, but the numerical routines would have to be imported from external libraries, which would have introduced additional dependencies.

\section{Acknowledgments}

We thank Johan Åkesson and Modelon AB for their initial and the continued development of the JModelica toolchain, and Joris Gillis and Joel Andersson for the development of CasADi, which were both instrumental for our toolchain. We also thank Joris Gillis, for his help and discussions about the implementation of our code using CasADi.

\section{Disclosure statement}

No potential conflict of interest was reported by the authors.

\section{Funding}

This work was supported by the Agency for Innovation by Science and Technology (IWT, Agentschap voor Innovatie door Wetenschap en Technologie) in Flanders, under grant No 131012.

\section{ORCID}

F. Jorissen (D) http://orcid.org/0000-0002-9273-0179

L. Helsen (D) http://orcid.org/0000-0002-9643-8204

\section{References}

HSL. 2017. "A Collection of Fortran Codes for Large-scale Scientific Computation.” http://www.hsl.rl.ac.uk/.

Åkesson, J., K.-E. Årzén, M. Gäfvert, T. Bergdahl, and H. Tummescheit. 2010. "Modeling and Optimization with Optimica and JModelica.org. Languages and Tools for Solving Large-scale Dynamic Optimization Problems." Computers \& Chemical Engineering 34 (11): 1737-1749. doi:10.1016/j.compchemeng.2009.11.011.

Andersson, J. A. E., J. Gillis, G. Horn, J. B. Rawlings, and M. Diehl. 2018. "CasADi - A Software Framework for Nonlinear Optimization and Optimal Control." Mathematical Programming Computation.

Andreas, W., and L. T. Biegler. 2006. "On the Implementation of an Interior-point Filter Line-search Algorithm for Large-scale Nonlinear Programming." Mathematical Programming 106 (1): 25-57.doi:10.1007/s10107-004-0559-y.

Bonvini, M., and M. Wetter. 2015. "Gradient-Based Optimal Control of Batteries and Hvac in District Energy Systems." 14th Conference of International Building Performance Simulation Association, Hyderabad, India.

Cagienard, R., P. Grieder, E. C. Kerrigan, and M. Morari. 2004. "Move Blocking Strategies in Receding Horizon Control." 43rd IEEE Conference on Decision and Control (CDC), Vol. 2, 2023-2028. doi:10.1109/CDC.2004.1430345.

Cigler, J., D. Gyalistras, J. Široký, V.-N. Tiet, and L. Ferkl. 2013. "Beyond Theory: the Challenge of Implementing Model Predictive Control in Buildings." 11 th REHVA world congress and 8th international conference Energy Efficient, Smart and Healthy Buildings, 1008-1018, Prague.

De Coninck, R., and L. Helsen. 2016. "Practical Implementation and Evaluation of Model Predictive Control for an Office Building in Brussels.” Energy \& Buildings 111: 290-298. doi:10.1016/j.enbuild.2015.11.014.

De Coninck, R., F. Magnusson, J. Åkesson, and L. Helsen. 2016. "Toolbox for Development and Validation of Greybox Building Models for Forecasting and Control." Journal of Building Performance Simulation 9: 288-303. doi:10.1080/19401493.2015.1046933.

Diehl, M. 2014. "Lecture Notes on Optimal Control and Estimation".

Diehl, M., H. Bock, H. Diedam, and P.-B. Wieber. 2006. Fast Direct Multiple Shooting Algorithms for Optimal Robot Control, 65-93. Heidelberg: Springer. ISBN 978-3-54036119-0.

Elmqvist, H., and S. E. Mattsson. 1997. "Modelica - The Next Generation Modeling Language - An International Design Effort." Proceedings of First World Congress of System Simulation, 1-5, Singapore.

Fritzson, P., P. Aronsson, H. Lundvall, K. Nyström, A. Pop, L. Saldamli, and D. Broman. 2005. "The OpenModelica Modeling, Simulation, and Software Development Environment." Simulation News Europe 44: 8-16.

Gebremedhin, A. H., F. Manne, and A. Pothen. 2005. "What Color is Your Jacobian? Graph Coloring for Computing Derivatives." SIAM Review 47 (4): 629-705.

Henze, G. P. 2013. "Model Predictive Control for Buildings: A Quantum Leap?.” Journal of Building Performance Simulation 66 (3): 157-158. 
Hilliard, T., M. Kavgic, and L. Swan. 2016. "Model Predictive Control for Commercial Buildings: Trends and Opportunities." Advances in Building Energy Research 10 (2): 172-190. doi:10.1080/17512549.2015.1079240.

International Energy Agency. 2015. "World Energy Outlook 2015." Technical report.

Jorissen, F., W. Boydens, and L. Helsen. 2018a. "Implementation and Validation of the Integrated Envelope, HVAC and Controller Model of the Solarwind Office Building in Modelica." Journal of Building Performance Simulation. (Submitted).

Jorissen, F., W. Boydens, and L. Helsen. 2018b. "TACO, an Automated Toolchain for Model Predictive Control of Building Systems: Application and Demonstration." Journal of Building Performance Simulation. (Submitted).

Jorissen, F., and L. Helsen. 2016. "Towards an Automated ToolChain for MPC in Multi-zone Buildings.” International High Performance Buildings Conference, West-Lafayette, IN. Paper 202.

Jorissen, F., G. Reynders, R. Baetens, D. Picard, D. Saelens, and L. Helsen. 2018. "Implementation and Verification of the IDEAS Building Energy Simulation Library." Journal of Building Performance Simulation. doi:10.1080/ 19401493.2018.1428361.

Nocedal, J., and S. J. Wright. 1999. Numerical Optimization: Springer Series in Operations Research, 65-93. Heidelberg: Springer-Verlag. ISBN 0-387-98793-2.

Picard, D., F. Jorissen, and L. Helsen. 2015. "Methodology for Obtaining Linear State Space Building Energy Simulation Models." 11th International Modelica Conference, 51-58, Paris, France. doi:10.3384/ecp1511851.

Prívara, S., J. Široký, L. Ferkl, and J. Cigler. 2011. "Model Predictive Control of a Building Heating System: The First Experience." Energy \& Buildings 43 (2-3): 564-572. doi:10.1016/j.enbuild.2010.10.022.

Široký, J., F. Oldewurtel, J. Cigler, and S. Prívara. 2011. "Experimental Analysis of Model Predictive Control for an Energy Efficient Building Heating System.” Applied Energy 88 (9): 3079-3087. doi:10.1016/j.apenergy.2011.03.009.

Sturzenegger, D., D. Gyalistras, M. Gwerder, C. Sagerschnig, M. Morari, and R. S. Smith. 2013. "Model Predictive Control of a Swiss Office Building.” 11th REHVA World Congress and 8th International Conference "Energy Efficient, Smart and Healthy Buildings", 3227-3236, Prague.

Sturzenegger, D., D. Gyalistras, M. Morari, and R. S. Smith. 2012. "Semi-automated Modular Modeling of Buildings for Model Predictive Control." Proceedings of the Fourth ACM Workshop on Embedded Sensing Systems for EnergyEfficiency in Buildings - BuildSys '12, 99-106, Toronto. doi:10.1145/2422531.2422550.

Sturzenegger, D., D. Gyalistras, M. Morari, and R. S. Smith. 2016. "Model Predictive Control of a Swiss Office Building: Implementation, Results, and Cost-Benefit Analysis." IEEE Transaction on Control Systems Technology 24 (1): 1-12. doi:10.1109/TCST.2015.2415411.

Sturzenegger, D., D. Gyalistras, V. Semeraro, M. Morari, and R. S. Smith. 2014. "BRCM Matlab Toolbox : Model Generation for Model Predictive Building Control." 2014 American Control Conference, 1063-1069, Portland.

Tarjan, R. 1972. "Depth-first Search and Linear Graph Algorithms." SIAM Journal on Computing 1 (2): 146-160. doi:10.1137/0201010.

Váňa, Z., J. Cigler, J. Široký, E. Záčeková, and L. Ferkl. 2014. "Model-based Energy Efficient Control Applied to an Office Building." Journal of Process Control 24 (6): 790-797. doi:10.1016/j.jprocont.2014.01.016.

Verhelst, J. 2017. "Fault Tolerance Assessment of HVAC-control in Office Buildings." PhD thesis, Arenberg Doctoral School, KU Leuven.

Wargorcki, P., O. Seppänen, H. Andersson, A. Boerstra, D. Clements-Croome, K. Fitzner, and S. Hanssen. 2006. "Indoor Climate and Productivity in Offices." REHVA. Guidebook No. 6.

West, S. R., J. K. Ward, and J. Wall. 2014. "Trial Results from a Model Predictive Control and Optimisation System for Commercial Building HVAC." Energy \& Buildings 72: 271-279. doi:10.1016/j.enbuild.2013.12.037.

Wetter, M., and C. van Treeck. 2017. "IEA EBC Annex 60: New Generation Computing Tools for Building and Community Energy Systems." The Regents of the University of California and RWTH Aachen University. ISBN 978-0-69289748-5. http://www.iea-annex60.org/pubs.html. 